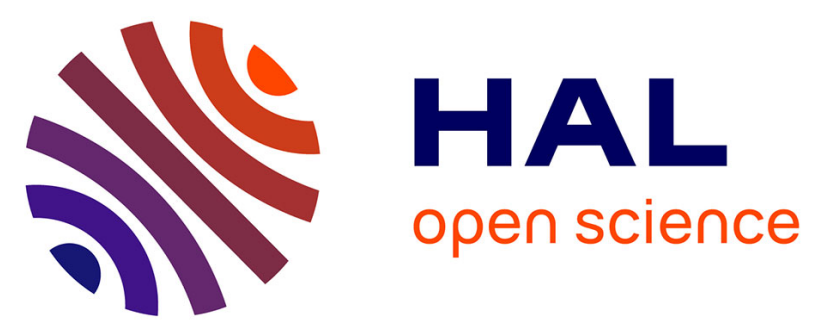

\title{
Multispectral reflectance imaging of brain activation in rodents: methodological study of the differential path length estimations and first in vivo recordings in the rat olfactory bulb
}

Rémi Renaud, Claire Martin, Hirac Gurden, Frederic Pain

\section{To cite this version:}

Rémi Renaud, Claire Martin, Hirac Gurden, Frederic Pain. Multispectral reflectance imaging of brain activation in rodents: methodological study of the differential path length estimations and first in vivo recordings in the rat olfactory bulb. Journal of Biomedical Optics, 2012, 17 (1), 10.1117/1.JBO.17.1.016012 . hal-01316107

\author{
HAL Id: hal-01316107 \\ https://hal.science/hal-01316107
}

Submitted on 14 May 2016

HAL is a multi-disciplinary open access archive for the deposit and dissemination of scientific research documents, whether they are published or not. The documents may come from teaching and research institutions in France or abroad, or from public or private research centers.
L'archive ouverte pluridisciplinaire HAL, est destinée au dépôt et à la diffusion de documents scientifiques de niveau recherche, publiés ou non, émanant des établissements d'enseignement et de recherche français ou étrangers, des laboratoires publics ou privés. 


\section{Biomedical Optics}

Multispectral reflectance imaging of brain activation in rodents: methodological study of the differential path length estimations and first in vivo recordings in the rat olfactory bulb

Rémi Renaud

Claire Martin Hirac Gurden Frédéric Pain 


\title{
Multispectral reflectance imaging of brain activation in rodents: methodological study of the differential path length estimations and first in vivo recordings in the rat olfactory bulb
}

Rémi Renaud, Claire Martin, Hirac Gurden, and Frédéric Pain

Université Paris-Sud, CNRS UMR8165, Orsay F-91405, France

\begin{abstract}
Dynamic maps of relative changes in blood volume and oxygenation following brain activation are obtained using multispectral reflectance imaging. The technique relies on optical absorption modifications linked to hemodynamic changes. The relative variation of hemodynamic parameters can be quantified using the modified Beer-Lambert Law if changes in reflected light intensities are recorded at two wavelengths or more and the differential path length (DP) is known. The DP is the mean path length in tissues of backscattered photons and varies with wavelength. It is usually estimated using Monte Carlo simulations in simplified semi-infinite homogeneous geometries. Here we consider the use of multilayered models of the somatosensory cortex $(\mathrm{SsC})$ and olfactory bulb (OB), which are common physiological models of brain activation. Simulations demonstrate that specific DP estimation is required for $\mathrm{SsC}$ and $\mathrm{OB}$, specifically for wavelengths above $600 \mathrm{~nm}$. They validate the hypothesis of a constant path length during activation and show the need for specific DP if imaging is performed in a thinned-skull preparation. The first multispectral reflectance imaging data recorded in vivo during OB activation are presented, and the influence of DP on the hemodynamic parameters and the pattern of oxymetric changes in the activated OB are discussed. $\odot 2012$ Society of Photo-Optical Instrumentation Engineers (SPIE). [DOI: 10.1117/1.JBO.17.1.016012]
\end{abstract}

Keywords: multispectral imaging; reflectance imaging; Monte Carlo simulations; olfactory bulb; somatosensory cortex; sensory activation; functional imaging.

Paper 11495P received Sep. 9, 2011; revised manuscript received Nov. 18, 2011; accepted for publication Nov. 18, 2011; published online Feb. 8, 2012.

\section{Introduction}

Intrinsic optical signal imaging (IOSI) is widely used to study brain sensory activation in animal models. It relies on local changes in total hemoglobin volume $(\Delta[\mathrm{HbT}])$ and relative changes in oxygenation following activation (changes in oxyhemogolobin concentration, $\Delta\left[\mathrm{HbO}_{2}\right]$ and changes in deoxyhemoglobin concentration, $\Delta[\mathrm{HbR}]$ ). These hemodynamic events translate into changes of the absorption of light by activated tissues. By simply shining light on exposed tissues and recording changes in reflected light intensity, it is possible to image sensory activation with high spatiotemporal resolution. ${ }^{1}$ The technique is relatively straightforward and inexpensive to implement ${ }^{2}$ because it requires only a scientific grade CCD, a stereozoom, and a stabilized light source. It has been used widely to study activation in the visual cortices, ${ }^{3,4}$ the somatosensory and barrel cortices ${ }^{5-8}$ and olfactory bulb (OB) ${ }^{9-11}$ The calculation of actual hemodynamic variations (oxy-, deoxy-, and total hemoglobin concentrations) from reflectance images requires recording at two or more wavelengths (multispectral imaging). A similar approach has been used extensively in humans in near-infrared spectroscopy (NIRS) and functional diffuse optical imaging. To date, multispectral imaging of the brain activation in rodents has been used to study somatosensory and barrel cortex activation under physiological and pathological

Address all correspondence to: Pain Frédéric, Université Paris Sud, Campus d'Orsay, Batiment 440, Laboratoire IMNC UMR8165 F-91405, Tel: +33169 1544 89, Fax: +33 1691571 96, E-mail: pain@imnc.in2p3.fr. conditions. ${ }^{8,12-16}$ To the best of our knowledge, however, multispectral recordings have not been reported in the $\mathrm{OB}$, despite the great interest of this structure in the study of neurovascular and neuroenergetics coupling following sensory stimulation. ${ }^{17}$ The OB contains remarkably well-defined functional modules, the olfactory glomeruli, which are easily accessible for functional optical imaging and can be stimulated by odorants at controlled physiological intensities and durations. ${ }^{9,10,17,18}$

The derivation of hemodynamic changes from reflectance images acquired at different wavelengths is based on the modified Beer-Lambert Law. This law depicts the attenuation of light in tissues and introduces the differential path length (DP) to take into account the dependence of photons path length on the wavelength in biological tissues. DP was shown to be a crucial corrective factor to derive relative variations in blood volume and hemoglobin oxygen saturation from reflectance variations ${ }^{19}$ but remains difficult to estimate accurately. ${ }^{20-22}$ The classic approach in small animal studies is to estimate it on the basis of Monte Carlo simulation (MCS) of backscattered photon travel in tissue according to the absorption, scattering, and anisotropic properties of tissues. ${ }^{8,23,24}$ Two major hypotheses are generally assumed when using DP estimates based on MCS and the modified Beer-Lambert Law to analyze multispectral data. First, MCSs are carried out in simplified homogeneous monolayer, semi-infinite geometries. Second, the DP is independent of time; that is, it remains constant during activation despite hemodynamic changes. 
We first considered these hypotheses in the context of somatosensory cortex $(\mathrm{SsC})$ and multispectral imaging. MCSs were carried out for both structures to evaluate the influence of the model's geometry on the estimated DP. The influence on DP of a thin bone layer remaining at the surface of the brain in a so -called thinned-skull preparation used in IOSI was also evaluated. In addition, the influence on DP of optical coefficient changes occurring during cerebral activation was estimated by varying absorption, scattering, and anisotropy independently. Second, the first multispectral recordings during OB activation are presented. In light of the findings from the MCSs, the effects of the DP used as correction factors on hemodynamic signal patterns are discussed.

\section{Materials and Methods}

\subsection{Modified Beer-Lambert Law}

In turbid media as biological tissues, the attenuation of light is caused by absorption and scattering which impose to consider a wavelength-dependent photon path length. ${ }^{19}$ The analysis of reflectance signals therefore relies on a modified Beer-Lambert Law:

$$
\frac{I_{\mathrm{det}, \lambda}(t)}{I_{\mathrm{inc}, \lambda}}=\exp \left[-\mu_{a, \lambda}(t) \mathrm{DP}_{\lambda}(t)+G(t)\right]
$$

where $I_{\text {inc }}$ is the incident light intensity, $I_{\operatorname{det}}$ is the detected intensity, DP is the wavelength-dependent path length, and $G$ is a factor representing the intensity loss caused by scattering. The absorption coefficient $\mu_{a, \lambda}$ (in $\mathrm{cm}^{-1}$ ) is the linear combination of the contribution of each chromophores and is rewritten as follows:

$$
\mu_{a, \lambda}(t)=\sum_{i} \ln 10 \times \varepsilon_{i, \lambda} \times c_{i}(t)
$$

where $\varepsilon_{i, \lambda}$ is the molecular extinction coefficient of each chromophore (in $l \cdot \mathrm{mol}^{-1} \cdot \mathrm{cm}^{-1}$ ) and $c_{i}$ is its concentration (in $\mathrm{mol} \cdot \mathrm{l}^{-1}$ ). In multispectral imaging of brain activation, the recorded data consist of successive images of reflected light intensity at several wavelengths before, during, and after activation. In Eq. (1), DP and $G$ are functions of time and are directly related to changes in tissues optical properties that occur following activation. The modifications of DP due to changes in optical properties during activation were evaluated in the present study (see Sec. 2.3). The validity of assuming a time-independent $G$ in the present study is discussed in Sec. 4. If both DP and $G$ can be considered constant following activation, the variation of light reflectance between rest and time $t$ after the onset in the stimulus can be computed at each wavelength as follows:

$$
\Delta A_{\lambda}(t)=-\log \left[\frac{I_{\text {detacti }, \lambda}(t)}{I_{\text {detrest }, \lambda}}\right]=\sum_{i} \varepsilon_{i, \lambda} \Delta c_{i}(t) \mathrm{DP}_{\lambda} .
$$

Assuming that changes in oxyhemoglobin $\left(\mathrm{HbO}_{2}\right)$ and deoxyhemoglobin (HbR) concentrations solely contributes to reflectance changes, the dynamic measurement of reflected intensity changes allows for determination of the time courses of $\Delta\left[\mathrm{HbO}_{2}\right]$ and $\Delta[\mathrm{HbR}]$.

\subsection{Geometry and Optical Properties of Somatosensory Cortex and Olfactory Bulb Models}

We performed MCS of photons path in monolayer and multilayer models of SsC and OB based on anatomical and physiological data gathered from the literature. ${ }^{25}$ The SsC multilayer model is composed of layers LI and LII (see Fig. 1). LI is $300 \mu \mathrm{m}$ thick and corresponds to L1 in the anatomical description of this cortex. LII is 2,700 $\mu$ m thick, and encompasses layers L2, L3, and L4, and is described by anatomical studies. Following sensory stimulation, L4 is activated and an electrical signal propagates upward to layers $\mathrm{L}_{2} / \mathrm{L}_{3}$ with in milliseconds. $^{26}$ The choice of $\mathrm{L} 4$ as the main activated layer in terms of hemodynamic changes (flow and oxygenation) is justified by the assumption that most of the energy needed to respond to activation is likely to be asked for in L4, where the signal initiates. This is supported by the high density of excitatory synapses, capillaries in L4 (for a review, see Ref. 26), and by the specific accumulation of $\left[{ }^{14} \mathrm{C}\right]$ 2-deoxyglucose (2DG) observed in autoradiographic experiments following stimulation of the somatosensory or barrel cortex. ${ }^{27}$ The SsC monolayer model is composed of a single semi-infinite layer bearing the optical properties of L4, which is the first activated layer. The OB multilayer model comprises three layers: the olfactory nerve layer (ONL, $140 \mu \mathrm{m}$ thick), the glomerular layer (GL, $100 \mu \mathrm{m}$ thick), and a layer that groups several anatomical layers (internal and external plexiform layers, mitral cell layer, and

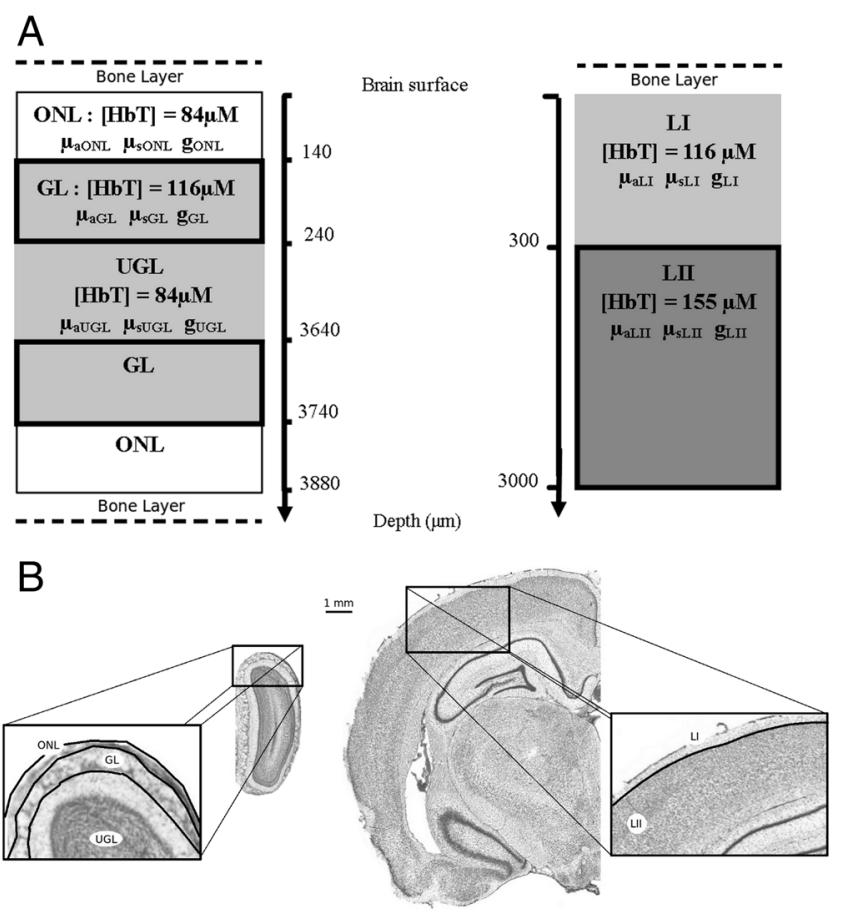

Fig. 1 Multilayer models for $\mathrm{SsC}$ and OB. (a) left: The OB model is composed of the ONL, the GL, and the UGL, which includes the external plexiform layer, the mitral cell layer, the internal plexiform layer, and the granular cell layer. (a) right: The SsC model is composed of layer LI and layer LII, including, respectively, layer L1 and layers L2, L3, and L4. The thick black lines show the layers where hemodynamic changes are expected to occur following activation (LII for SsC and GL for OB). The dashed black lines show the model with a superficial bone layer. (b) OB (left) and $\mathrm{SsC}$ (right) coronal sections with magnification of imaged layers are shown. 
Table 1 Wavelength-dependent optical properties used for the bone layer in MCS.

\begin{tabular}{|c|c|c|c|c|c|c|c|c|c|c|c|c|c|}
\hline Wavelength $(\mathrm{nm})$ & 440 & 460 & 487 & 492 & 530 & 560 & 570 & 584 & 610 & 630 & 671 & 700 & 730 \\
\hline$\mu_{a}\left(\mathrm{~cm}^{-1}\right)$ & 1.30 & 0.90 & 0.90 & 0.90 & 0.70 & 0.70 & 0.70 & 0.70 & 0.65 & 0.60 & 0.45 & 0.45 & 0.40 \\
\hline$\mu_{s}\left(\mathrm{~cm}^{-1}\right)$ & 280 & 250 & 230 & 230 & 225 & 225 & 225 & 225 & 210 & 200 & 200 & 200 & 190 \\
\hline$g$ & 0.9 & 0.9 & 0.9 & 0.9 & 0.9 & 0.9 & 0.9 & 0.9 & 0.9 & 0.9 & 0.9 & 0.9 & 0.9 \\
\hline
\end{tabular}

granular cell layer) called the underneath the glomerular layer (UGL, 3,400 $\mu \mathrm{m}$ thick). Symmetrically inverted layers relative to the UGL were considered to take into account the symmetry of the OB. Because previous autoradiographic and intrinsic optical imaging studies by our group and others have shown 2DG accumulation and reflectance changes localized in spotty areas identified anatomically as glomeruli (for a review, see Ref. 17), the OB monolayer model is composed of a single semiinfinite layer bearing the optical properties of the GL.

The absorption coefficient $\mu_{a, \lambda}$ is wavelength-dependent homogeneous for each layer. This coefficient accounts for absorption due to total hemoglobin $\left(\mu_{a-\mathrm{HbT}, \lambda}\right)$ and minor, but not negligible, absorption from other absorbers $\left(\mu_{a \text {-cell, }, \lambda}\right)$ as detailed previously: ${ }^{25}$

$$
\mu_{a, \lambda}=\mu_{a-\mathrm{HbT}, \lambda}+\mu_{a \text {-cell }, \lambda} .
$$

Absorption coefficients for hemoglobin at different wavelengths $\left(\mu_{a-\mathrm{HbT}, \lambda}\right)$ were calculated for the different layers using tabulated molar extinction coefficients for oxy- and deoxy-hemoglobin. ${ }^{28}$ Oxygen saturation at rest was set to $60 \%(S=0.6) .^{23,29}$ The absorption coefficient for other absorbers $\left(\mu_{a \text {-cell, } \lambda}\right)$ was taken from experimental measurements in tissues devoid of blood. ${ }^{30}$ In addition, a wavelengthindependent scattering coefficient, $\mu_{s}$, and a wavelengthdependent anisotropy factor, $g$, were considered for each layer. Scattering coefficients and anisotropy factors were taken from measurements on white and gray matters in blood-free human brain tissues. ${ }^{30}$ The refractive index $n$ for tissue was fixed at 1.40 for all layers. ${ }^{31-33}$

\subsection{Monte Carlo Simulations and Differential Path Length Calculation}

MCSs of photon travel in tissues are based on validated algorithms ${ }^{34,35}$ that were custom-implemented. We performed MCSs for anatomo functional models of SsCs and OBs to estimate DP at wavelengths from 440 to $730 \mathrm{~nm}$. DP obtained in monolayer geometry with infinite depth were compared to DP calculated using multilayer geometry with finite depth. In homogeneous geometry, the "differential path length" is defined as the partial derivative of absorbance by the absorption coefficient:

$$
\mathrm{DP}_{\lambda}=\frac{\partial A_{\lambda}}{\partial \mu_{a, \lambda}} .
$$

In a multilayer model, the practical use of this definition to evaluate DP from MCS is not straightforward. The generalization of the modified Beer-Lambert Law to heterogeneous models requires introduction of the partial path length for the $i$ 'th layer, defined as $\partial A / \partial \mu_{a i}$. The derivatives, which are dependent upon optical properties, are calculated for each layer from the MCS, thus allowing differential spectroscopic data analysis to be performed. This approach was evaluated in initial sets of experimental data and compared with the approach using the mean path length throughout the tissue as DP. It was found that although not mathematically rigorous, calculating the DP as the mean path length traveled through the layered model led to results similar to those derived by using partial path lengths. This approximation was much more simple to implement and was consequently used for further analysis.

In a first set of simulations, the DPs obtained for the $\mathrm{SsC}$ and $\mathrm{OB}$ in monolayer and multilayer geometries were compared. In a second set of stimulations, the influence on DP of changes in the optical properties occurring during activation was assessed in the OB model. Each optical parameter was varied independently in a global manner (all layers) or locally (in the glomerular layer). Changes of $+10 \%$ of $\mu_{a, \lambda} \pm 10 \%$ of $\mu_{s}$ and $+5 \%$ of $g$ were simulated. In a third set of simulations, the effect of a superficial bone layer on the DP was studied. A bone layer thickness of $50 \mu \mathrm{m}$ was simulated, which corresponds to the experimental paradigm in which images of the brain are acquired through the thinned skull to limit invasiveness and potential tissue alterations. Bone optical properties vary with wavelength as described previously in the literature ${ }^{36,37}$ and are given in Table 1.

Depending on the optical parameters and the number of layers considered, it took from less than 1 minute up to 15 minutes to propagate $10^{6}$ - photon packets using a personal computer with 8 Go RAM and a dual core $2.7 \mathrm{GHz}$ processor.

\subsection{Intrinsic Signal Optical Imaging}

\subsubsection{Animal preparation}

All studies were conducted in accordance with European Commission directive 2010/63/EU. Long Evans rats (mean weight $=300 \mathrm{~g}$ ) were anesthetized with an intraperitoneal injection of ketamine $(60 \mathrm{mg} / \mathrm{kg}$ Imalgen, ) and metedomidin $(0.4 \mathrm{mg} / \mathrm{kg}$ Domitor) mix. The anesthesia level was monitored and adjusted if necessary throughout the experiment. Rats were then placed in a stereotactic instrument. A cranial window of about $3 \times 3 \mathrm{~mm}^{2}$ was created over the OB and filled with agarose covered by a microscope coverslip to minimize brain movements and ensure a flat optical interface. Although imaging through thinned bone was considered in the DP study, all experimental studies (three rats) were carried out with a cranial window with the cranial bone removed. Body temperature was monitored and maintained with a heating pad throughout the experiment.

\subsubsection{Imaging setup and acquisition session}

Reflectance images evoked by OB activation were recorded with a cooled 12-bits CCD camera (CoolSNAP ES ${ }^{2}$; Photometrics, Tucson, AZ, USA) operated at 4-by-4 binning and mounted 
on a Leica stereomicroscope (MZ16 FA; Leica Microsystems, Wetzlar, Germany). The illumination was performed using a light engine (OneLight Spectra VISX; OneLight Corp., Canada) based on a broad-band light source and a digital mirror device (DMD; Texas Instruments,Dallas, TX, USA). Light passes through a grating (600-lpm grating), is scattered on the DMD, composed of $1,024 \times 768$ mirrors. The index of the columns of open mirrors defines the transmitted wavelength band, and the number of open rows sets the intensity. Spectral output ranging from $430 \mathrm{~nm}$ to $680 \mathrm{~nm}$ can be set with a spectral resolution of 5-nm FWHM, which was measured using a fibered spectrophotometer (AvaSpec-2048, ×14; Avantes, Inc., Broomfield, CO, USA). Moreover, the light engine can store eight mirror patterns prior to the experiment, which allows fast switching between wavelengths (switch is achieved in less than $40 \mu \mathrm{s}$ ) and illuminates the tissue at three images/wavelength/second. Stimulation of the OB was performed with pure odorants (hexanal diluted at $10 \%$ in mineral oil) delivered by a transistortransistor logic (TTL)- controlled olfactometer (Pressurized Superfusion, AutoMate Scientific Berkeley, CA, USA). The CCD camera and olfactometer were controlled using a custom - written $\mu$ Manager script. ${ }^{38}$ The light source was operated in slave mode using a custom LabVIEW System Design software program (National Instruments, Austin, TX, USA) and waits for a trigger from the CCD camera. The camera issues an exposure out TTL signal, which is sent to the lamp and used to switch to the next wavelength pattern. Brain reflectance under green light $(530 \mathrm{~nm})$ is first recorded to visualize blood vessels architecture. Then, for reflectance imaging, illumination is carried out sequentially at 440, 487, 530, 560, 584, 610,630, and $671 \mathrm{~nm}$ during the imaging trial, which consists of $5 \mathrm{sec}$ of rest, $5 \mathrm{sec}$ of odor stimulation by hexanal $10 \%$, and $50 \mathrm{sec}$ post stimulation. The intertrial interval is at least $1 \mathrm{~min}$ to avoid habituation of the rat to the stimulus. Images are acquired at a frame rate of $3 \mathrm{~Hz}$ per wavelength (24 frames per second). Further details regarding the timing of the imaging set-up and image processing can be found in. ${ }^{39,40}$

\subsubsection{Image processing}

Each stimulation trial produces 16-bit raw movies of $\mathrm{OB}$ reflected intensities for each wavelength, gathering prestimulation, stimulation, and poststimulation periods. To derive 32-bit reflectance variation movies, each frame is normalized to the baseline frame (mean image of the 5-s prestimulation period). The modified Beer-Lambert analysis [Eq. (3)] is then fitted to the experimental data to obtain $\Delta\left[\mathrm{HbO}_{2}\right]$ and $\Delta[\mathrm{HbR}]$ dynamic maps. $\Delta[\mathrm{HbT}](t)$ maps are the sum of $\Delta\left[\mathrm{HbO}_{2}\right](t)$ and $\Delta[\mathrm{HbR}](t)$ at each time point.

\section{Results}

\subsection{Differential Path Length Estimation in Mono-versus Multilayer Geometries}

DP was calculated in $\mathrm{OB}$ and $\mathrm{SsC}$ for mono- and multilayer geometries at basal conditions without any sensory activation. As shown in Fig. 2, the DP for both structures were similar for wavelengths below $600 \mathrm{~nm}$ for all models (OB mono- and multilayer as well as SsC mono- and multilayer). For higher wavelengths, DPs in the OB were significantly higher than DPs in the SsC cortex. Consideration of layered models leads to a decrease in DP above $600 \mathrm{~nm}$ for both structures.

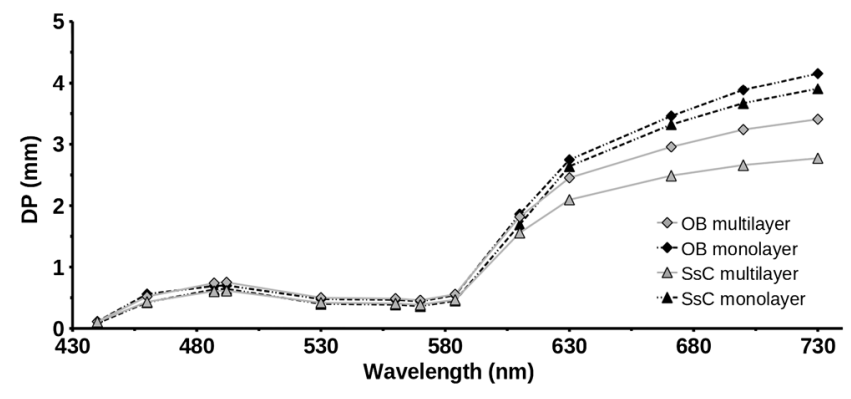

Fig. 2 Differential path length estimation in mono- and multilayer geometries under basal conditions for $\mathrm{OB}$ and $\mathrm{SsC}$ models. Light gray diamonds: $\mathrm{OB}$ multilayer 3.88-mm- thick model; black diamonds: OB monolayer 100-mm-thick model; black triangles: SsC monolayer 100-mm-thick model; light gray triangles: SsC multilayer 3-mmthick model.

The magnitude of the decrease relative to the DP calculated in monolayer models was similar for both structures and ranged between $\sim 10 \%$ at $630 \mathrm{~nm}$ and $\sim 25 \%$ at $730 \mathrm{~nm}$.

\subsection{Effect of Optical Properties Variations on Differential Path Length Estimation for Olfactory Bulb Multilayer Model}

The effects on DP of the variations of absorption, scattering, and anisotropy during tissue functional activation were investigated in the OB multilayered geometry. These results are summarized in Fig. 3. For a $10 \%$ local increase of the absorption coefficient, no changes in DP were observed below $600 \mathrm{~nm}$ [Fig. 3(a)]. For higher wavelengths, a decrease ranging from $-3 \%$ at $671 \mathrm{~nm}$ up to $-6 \%$ at $730 \mathrm{~nm}$ was observed,. Similar results were obtained when a global increase of absorption was considered, which lead to a decreases from $-4 \%$ at $630 \mathrm{~nm}$ up to $-8 \%$ at $730 \mathrm{~nm}$ [Fig. 3(a)]. DP was then calculated taking local and extended variations of the scattering coefficient into consideration [Figs. 3(b) and 3(c)]. Local and extended decreases of the scattering coefficient did not produce changes in the DP, except for wavelengths above $700 \mathrm{~nm}$. Local increases in scattering lead to a decrease of DP only for wavelengths above $670 \mathrm{~nm}(-4 \%$ at $671 \mathrm{~nm}$ and $-6.5 \%$ at $700 \mathrm{~nm}$ and $730 \mathrm{~nm}$ ). A similar tendency was observed when an extended increase of the scattering coefficient was taken into consideration $(-3 \%$ at $630 \mathrm{~nm},-6 \%$ at $671 \mathrm{~nm}$ and $-8 \%$ at 700 and $730 \mathrm{~nm})$. The effect of a 5\% increase of the anisotropy factor was also simulated [Fig. 3(d)]. No significant differences were apparent when the DP at basal conditions and the DP for a localized increase in anisotropy factor were compared, except at $730 \mathrm{~nm}(+5 \%)$. When a global change was simulated, a marked increase of DP was observed for the whole wavelength range from $+5 \%$ at $530 \mathrm{~nm}$ and $30 \%$ at $630 \mathrm{~nm}$ down to $+13 \%$ at $730 \mathrm{~nm}$ [Fig. 3(d)].

\subsection{Effect of a Superficial Bone Layer on Differential Path Length Estimation in Multilayer Olfactory Bulb Model}

Intrinsic optical imaging performed on anesthetized rats requires local removal or thinning to transparency of the skull to obtain a cranial window. A $50-\mu \mathrm{m}$-thick bone layer produces a moderate increase in the DP along the whole wavelength interval (Fig. 4). The relative increase ranges from $12 \%$ at $460 \mathrm{~nm}$ up to $18 \%$ at $630 \mathrm{~nm}$. The absolute increase is stronger for higher wavelengths, with increases up to $400 \mu \mathrm{m}$ for $700 \mathrm{~nm}$ and $730 \mathrm{~nm}$. 

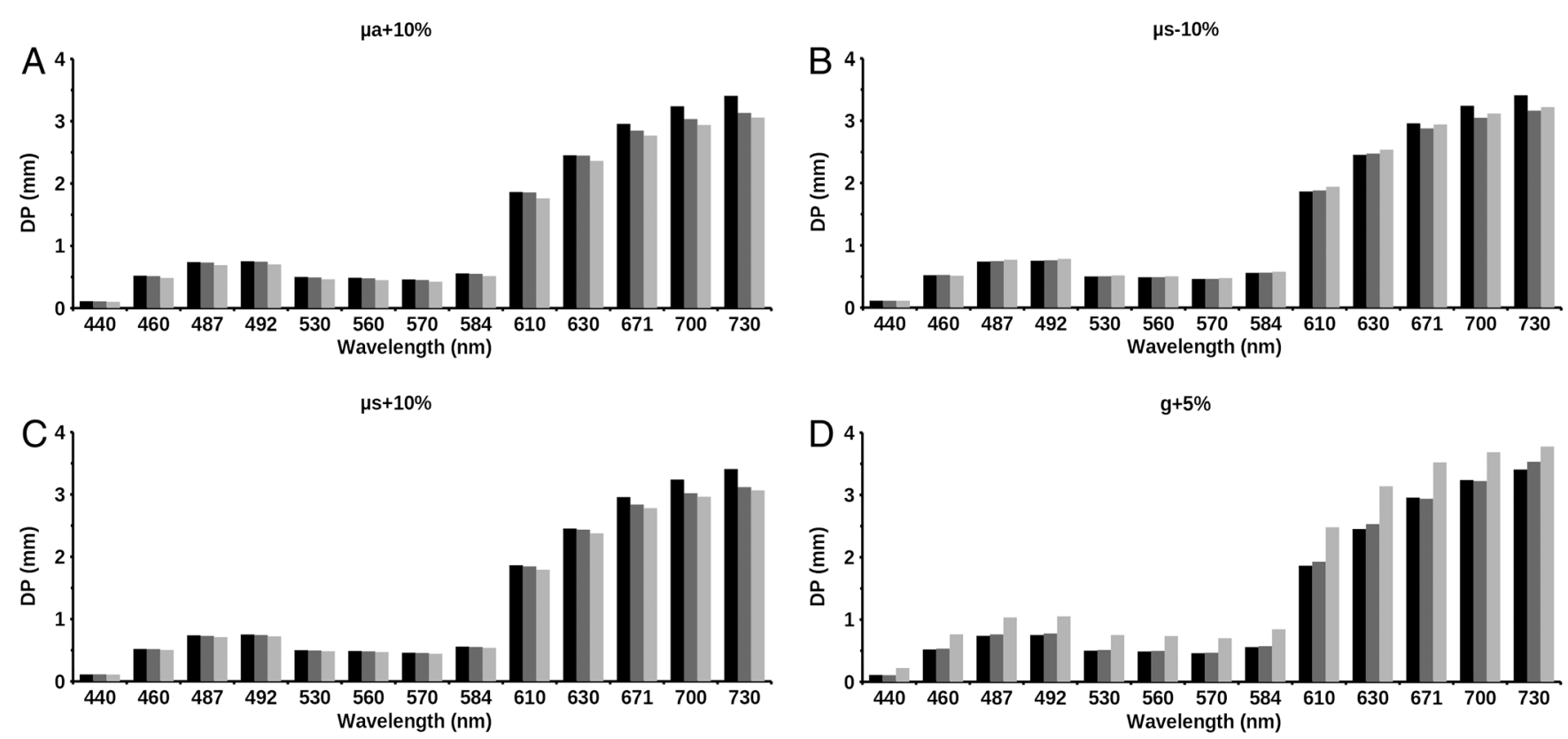

Fig. 3 DP estimation for optical coefficient variation in the OB multilayer model. (a) $10 \%$ increase of the absorption coefficient; (b) $10 \%$ decrease of the scattering coefficient; (c) $10 \%$ increase of the scattering coefficient; (d) $5 \%$ increase of the anisotropy factor. For all graphs,: black bars: DP at basal conditions; dark gray bars: DP for optical modifications in the glomerular layer only; and light gray bars: DP for extended variation (all layers).

\subsection{Contribution of Each Layer to the Global Path Length}

Using an OB multilayer model, we investigated the maximum depth reached by photons that are ultimately backscattered and

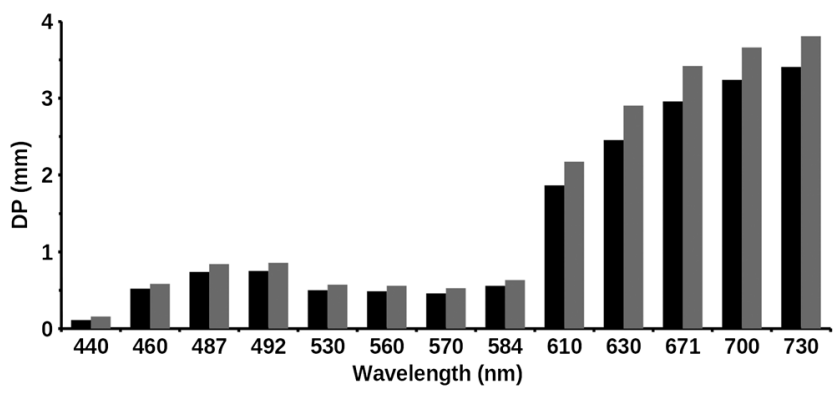

Fig. 4 Influence of a superficial bone layer on DP in the OB multilayer model. Black bars: no bone; dark gray: $50-\mu$ m-thick bone layer. the relative paths traveled in each layer. The maximum depth reached by photons ultimately detected was strongly dependent on wavelength. As shown in Fig. 5(a), only $10 \%$ of the reflected photons reached a depth of about $1.1 \mathrm{~mm}$ at $530 \mathrm{~nm}$ and $584 \mathrm{~nm}$, whereas at $630 \mathrm{~nm}, 10 \%$ of the reflected photons travel down to $1.7 \mathrm{~mm}$ from the brain surface. If we focus on the "activated layer" (i.e., the glomerular layer between 140 and $240 \mu \mathrm{m}$ deep), $20 \%$ of 530-nm and 584-nm photons exit the tissue without penetrating the glomerular layer. The higher absorption of blood at these wavelengths compared to $630 \mathrm{~nm}$ caused a larger amount of photons to be stopped in the glomerular layer and deeper within it. Figure 5(b) presents the relative path length traveled by photons in each layer expressed as percentages of the total path length. Backscattered photons principally propagated in UGL and traveled for a short distance in the superficial olfactory nerve layer (ONL). About $30 \%$ of the total path length occured in the GL for wavelengths shorter than $600 \mathrm{~nm}$ and about $20 \%$ for wavelengths above, despite longer overall path length at higher wavelengths.
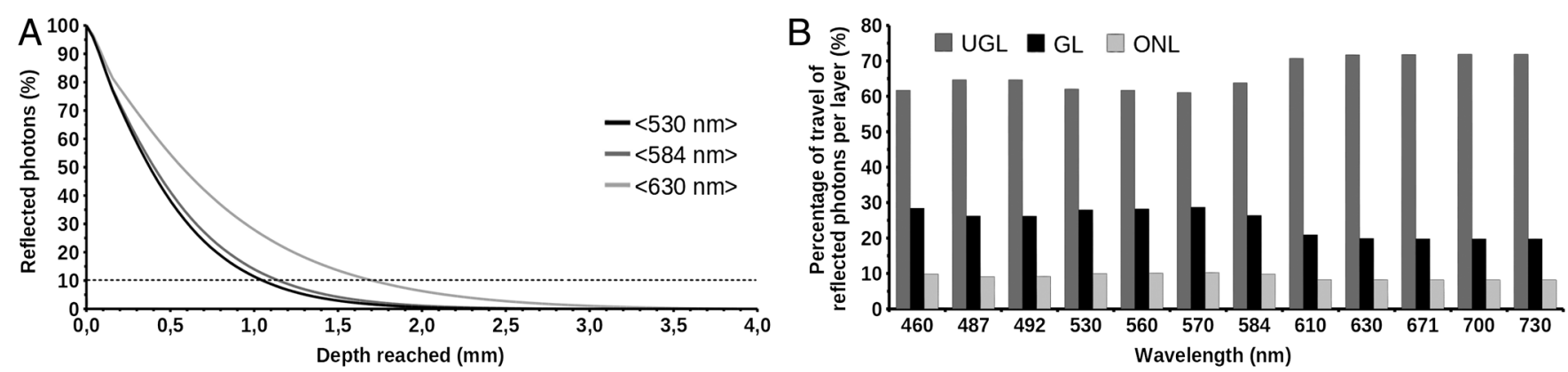

Fig. 5 Depths reached by photons in the OB multilayer model and contribution of each layer to the total DP. (a) Percentage of exiting photons as a function of depth reached within tissues. Black line: photons at $530 \mathrm{~nm}$; dark gray line: $584 \mathrm{~nm}$; and clear gray line: $630 \mathrm{~nm}$. Dashed line corresponds to the depth reached by $90 \%$ of reflected photons. (b) Percentage of total path traveled in each layer for exiting photons. Black bars: percentage of path in GL; dark gray bars: percentage of path in UGL; and light gray bars: percentage of path in ONL. 


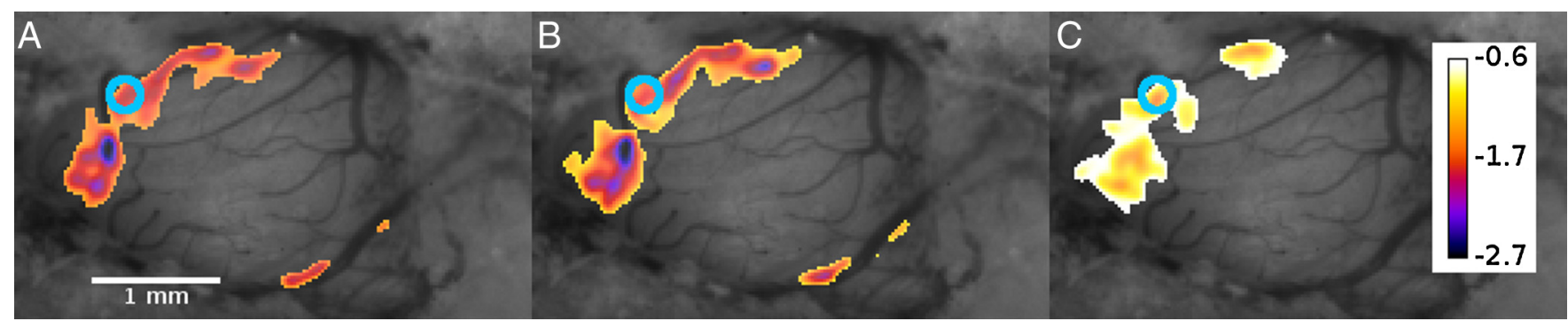

Fig. 6 Typical activation maps obtained at 530, 584, and $630 \mathrm{~nm}$ superimposed on a vascular map at $530 \mathrm{~nm}$. A threshold at $30 \%$ of maximum intensity of activation was applied to activation maps prior to superimposition. (a) activation map at $530 \mathrm{~nm}$; (b) activation map at $584 \mathrm{~nm}$; (c) activation map at $630 \mathrm{~nm}$. The color scale applies to all three images and stands for relative variation of reflectance from basal state (\%). The blue circle corresponds to the ROI used to derive hemodynamic time courses.

\subsection{Influence of Differential Path Length on Hemody- namic Parameters Calculation for Ob Activation}

A fast multispectral imaging set up was built, taking advantage of a DMD spectral light source with wavelength switch-time below $1 \mathrm{~ms}$. It allowed the recording of the first multispectral data during sensory activation of the $\mathrm{OB}$ in the anesthetized rat. A data cube of 348 by 260 pixels by 8 wavelengths was acquired at a rate of 3 full cube/s. The time courses of reflectance variation at each wavelength were derived from a region of interest (ROI) defined over the activated area.

Figure 6 presents typical activation maps obtained at $530 \mathrm{~nm}$, $584 \mathrm{~nm}$, and $630 \mathrm{~nm}$ in one rat during the same stimulation trial. For all wavelengths a marked decrease of reflectance was measured in the same OB area, although the activated zone did not entirely overlap.

The time courses of the relative reflectance changes obtained for $10 \%$ hexanal stimulation are presented for three wavelengths in Fig. 7(a). For the sake of clarity, the time courses recorded during three identical olfactory stimulations in one animal were averaged to remove part of the low - frequency oscillations of reflected light due to respiratory motion. For all wavelengths a sustained decrease in the intensity of reflected light occurred within 1 to $2 \mathrm{sec}$ poststimulation onset. Maximum relative changes were observed at the end of the stimulation duration and varied from $0.1 \%$ to $3 \%$, depending on the wavelength. The return to baseline was faster at $630 \mathrm{~nm}$ (about 20 to $30 \mathrm{sec}$ ) compared to 530 and $584 \mathrm{~nm}$ (about $40 \mathrm{sec}$ ). The time courses of $\Delta[\mathrm{HbT}], \Delta\left[\mathrm{HbO}_{2}\right]$, and $\Delta[\mathrm{HbR}]$ in the same ROI were derived from the raw reflectance signals at 530 and $630 \mathrm{~nm}$ by using the modified Beer-Lambert Law and DP factors at basal state for the multilayer OB model [Fig. 7(b)]. A moderate increase in the total blood volume occured following stimulation (maximum amplitude change $+4 \%$ at $5 \mathrm{sec}$ poststimulation) with a return to baseline about $20 \mathrm{sec}$ after the end of the stimulation. Regarding oxygenation changes, a large increase in $[\mathrm{HbR}]$ (up to $12 \%$ ) occurred with a return to baseline about $20 \mathrm{sec}$ after the end of the stimulation. These time courses were compared to those obtained with DP derived from the monolayer OB model and also to those obtained without taking into consideration the wavelength-dependent path length. In the latter case, the same DP value was applied to data acquired at all wavelengths (Fig. 8). The choice of the DP model did not affect the general temporal patterns of $\Delta[\mathrm{HbT}]$,or $\Delta[\mathrm{HbR}]$ but did have a strong impact on their amplitude. The use of a multilayer model of OB instead of a monolayer model led to a twofold decrease in the amplitude of $\Delta[\mathrm{HbT}]$ over the whole activation period and slightly decreased the maximum amplitude of $\Delta[\mathrm{HbR}]$. Furthermore, the return to baseline of $\Delta[\mathrm{HbR}]$ was delayed by about 5 to $10 \mathrm{sec}$. The use of the DP derived from the $\mathrm{SsC}$ monolayer model (data not shown) led to similar effects for both $\Delta[\mathrm{HbR}]$ and $\Delta[\mathrm{HbT}]$ : a large increase of amplitude for the whole time course of $\Delta[\mathrm{HbT}]$, and a moderate increase of maximum amplitude and time shift for $\Delta[\mathrm{HbR}]$. Using no wavelength-dependent DP correction, at least one
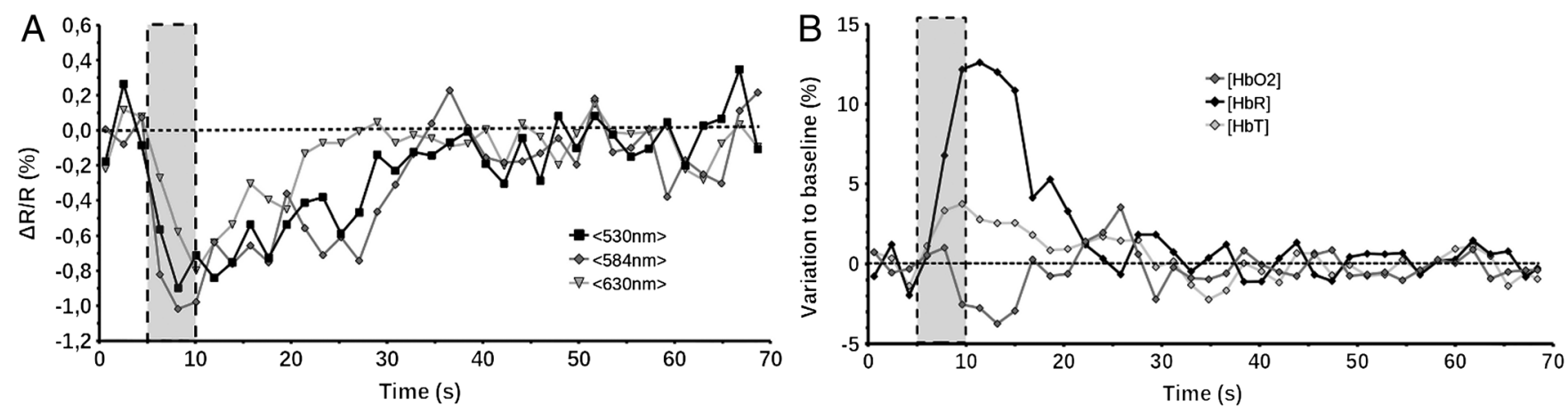

Fig. 7 Time course of optical reflectance changes and corresponding hemodynamic parameters changes during olfactory stimulation experiments. (a) Time course of reflectance changes relative to baseline for illumination at $530 \mathrm{~nm}$ (black squares), $584 \mathrm{~nm}$ (black diamonds) and $630 \mathrm{~nm}$ (grey triangles). Time courses of reflectance changes were derived on a ROI defined over an activated area devoid of large blood vessels [see blue circle in Fig. 6(c)]. (b) Time course of relative changes in Oxy-hemoglobin ( $\mathrm{HbO}$, dark grey diamonds), Deoxy-hemoglobin (Hbr, black diamonds) and Total hemoglobin ( $\mathrm{Hbt}$, light grey diamonds) concentrations. Time course of hemodynamic changes were derived using the modified Beer Lambert law and DP factors at basal state for the multilayer OB model. On both graphs, grey rectangles correspond to the 5 sec delivery of odorant stimulus. To remove part of the noise due to respiratory motion, time courses were averaged over three independent stimuli recorded successively in the same animal. 

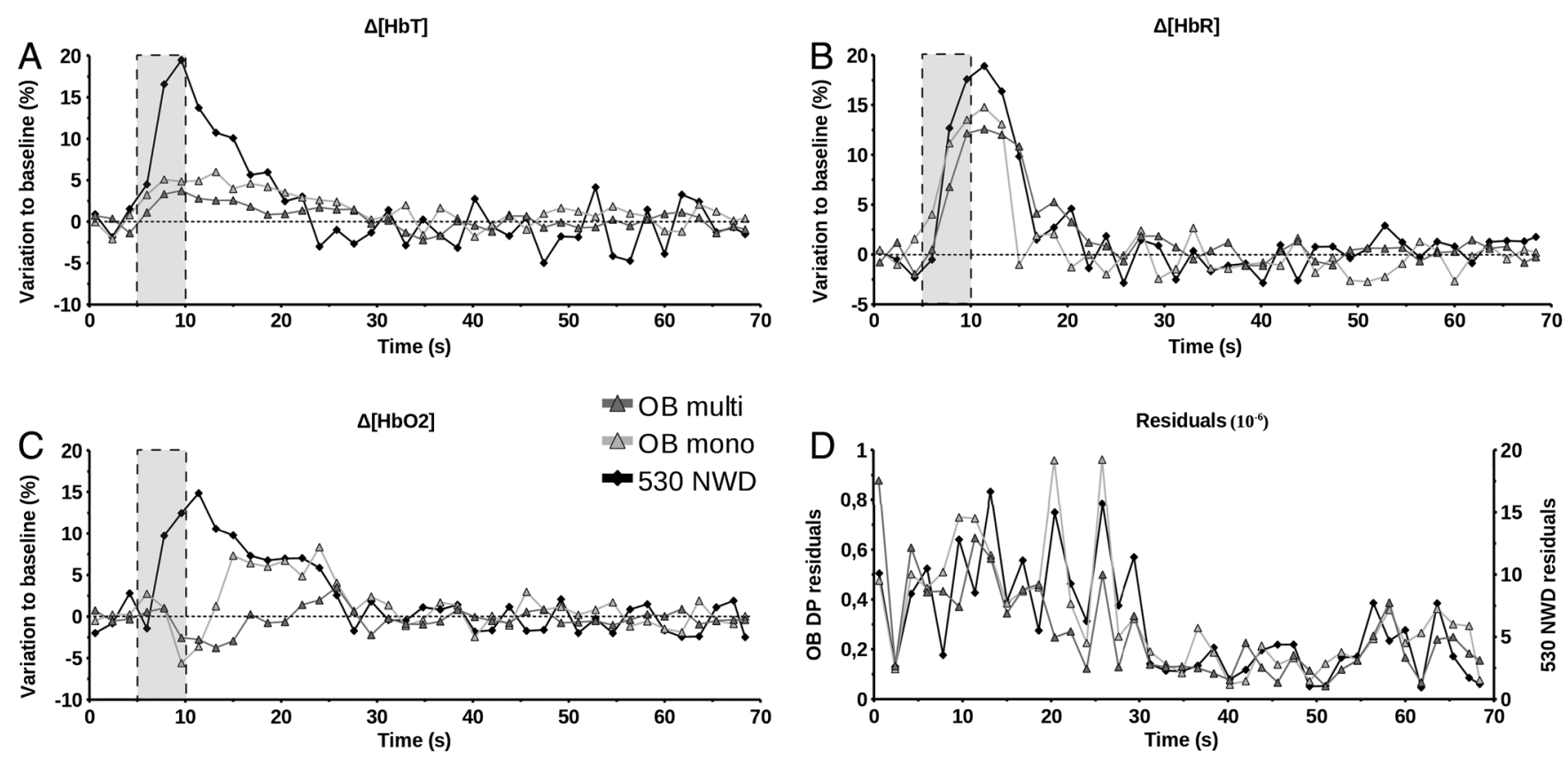

Fig. 8 Influence of the DP factors on the derivation of the hemodynamic parameters. Three models were considered to derive the hemodynamic parameters from the raw reflectance images. The DP used in the modified Beer Lambert calculation were (i) those simulated using the OB monolayer model, (ii) those simulated using the OB multilayer model or (iii) no pathlength dependant factor in which case the DP estimated at $530 \mathrm{~nm}$ was used for all wavelengths. On graphs (a)-(c), the large grey rectangle correspond to the delivery of odorant stimulus between $t=5 \mathrm{sec}$ and $t=10 \mathrm{sec}$. To remove part of the noise due to respiratory motion, time courses were averaged over three independent stimuli recorded successively in the same animal. (a) Influence of the DP factor on the total hemoglobin concentration relative changes. (b) Influence of the DP factor on the Deoxy-hemoglobin concentration relative changes. (c) Influence of the DP factor on the Oxy-hemoglobin concentration relative changes. (d) Fit residuals. Left $Y$ scale applies for fits using monolayer and multilayer models. Right $Y$ scale applies for the fit using no pathlength correction. For all graphs: grey triangles indicate that the DP from the OB multilayer model were used, light grey triangles indicate that the DP from the OB monolayer model were used, and black diamonds indicate that no pathlength correction was used.

of the parameters relative changes was over- or underestimated compared to the wavelength-dependent analysis. For instance, using the DP at $530 \mathrm{~nm}$ for all wavelengths led to a large overestimation of $\Delta[\mathrm{HbT}]$, whereas using the DP at $630 \mathrm{~nm}$ led to a large underestimation of $\Delta[\mathrm{HbR}]$. Estimation of $\Delta\left[\mathrm{HbO}_{2}\right]$ was rather more sensitive to which DP is used compared to $\Delta[\mathrm{HbR}]$ or $\Delta[\mathrm{HbT}]$. If the OB multilayer model is chosen, a transient decrease in oxygenation was observed that reached its maximum $(-4 \%)$ a few seconds after the end of the stimulation, followed by a return to baseline at about $20 \mathrm{sec}$ post stimulation. If a monolayer model for either $\mathrm{OB}$ or $\mathrm{SsC}$ is chosen, a lower decrease in $\Delta\left[\mathrm{HbO}_{2}\right]$ was observed and was followed by a delayed increase in $\Delta\left[\mathrm{HbO}_{2}\right]$ up to $7 \%$ at $25 \mathrm{sec}$ post stimulation. When no wavelength-dependent correction was taken into consideration, a moderate (using DP at $630 \mathrm{~nm}$ ) or strong (using $\mathrm{DP}$ at $530 \mathrm{~nm}$ ) increase in $\Delta\left[\mathrm{HbO}_{2}\right]$ occured within the stimulation period, with a return to baseline at $25 \mathrm{sec}$ poststimulation.

\section{Discussion}

DP has been acknowledged for a long time as an important correction factor to derive relative changes in oxymetric parameters from reflectance imaging at several wavelengths. ${ }^{20,41}$ Incorporation of this physical factor has been shown to strongly influence the analysis of reflectance measurements, especially with regard to the initial dip controversy debated within the neurovascular coupling community. ${ }^{42-44}$ Surprisingly, models used to calculate or estimate DP have remained relatively coarse, with the major approaches being, respectively, (1) the direct measurement of a solution of blood and diffusers ${ }^{21,22,45}$ (intralipid or polymeric spheres) or (a) a simple semi-infinite monolayer model for MCSs. In previous studies in humans, researchers have analyzed the influence of the heterogeneity and multilayered structure of brain cortical tissues. ${ }^{46-48}$ These studies have shown that, based on NIRS, the DP increases linearly with the thickness of the scalp. However, DP is relatively insensitive to the thickness of other layers. ${ }^{47}$ In another study, the cerebrospinal fluid layer was found to confine the light to the upper layer of the brain and increase DP. Differences in DP calculated in layered and complex models were found to be slight, even for IR illumination, owing to the dimensions of the human brain layers that exceed the mean path length of photons. ${ }^{49}$ For in vivo studies of rodents, the influence of the geometry has been found to be potentially much more crucial because the dimensions of the tissue layers are much smaller. Consequently, it is clear that even when visible light is used, several brain layers with distinct optical properties contribute to the reflectance changes. To explore the influence on the DP of anatomical (heterogeneity between layers) and physiological changes (absorption and scattering changes due to activation), we have carried out MCS for two rat brain structures: $\mathrm{SsC}$ and $\mathrm{OB}$. Both structures have proven to be consistent models with which to study brain activation as well as neurovascular and neuroenergetic coupling.

Multilayer models used for MCS have been developed previously in our laboratory. ${ }^{25}$ Although such models are, by nature imperfect in the absence of measured optical properties, they are based on rigorous analysis of the literature and realistic assumptions regarding tissue structures and physiology. The optical parameters for each layer have been set according to the anatomofunctional data gathered from the literature as detailed and 
discussed previously. ${ }^{25}$ One major criticism of the MCS models that could be made regards the homogeneity of cortical layers, due to the large blood vessels in the upper layers. Localized absorption in blood vessels has been addressed previously in MCS models ${ }^{50}$ and the use of actual expression to evaluate the corrected absorption and scattering coefficients has been proposed. ${ }^{51}$ Homogenization based on the optical properties weighted with their volume fractions is valid, while the diameters of vessels multiplied by the interaction coefficient $\left(\left[\mu_{, t}=\mu_{a}+\mu_{s}(1-g)\right]\right.$ is below unity. Talsma et al ${ }^{51}$ proposed the derivation of the apparent absorption coefficient regarding the diameter of the blood vessels. Because most vessels in the GL and UGL are capillaries or arterioles and venules with small diameters, the assumption of homogeneity in these layers can be made.

The calculations of DP in multilayer finite versus monolayer infinite geometries showed (1) a significant decrease of the DP for wavelengths above 600 to $630 \mathrm{~nm}$ for both brain structures and (2) substantially lower DP in the SsC compared to the OB for both models (Fig. 2). Decreases in the DP for wavelengths above 600 to $630 \mathrm{~nm}$ in multilayer versus monolayer models is essentially due to the finite thickness of multilayer models. If finite monolayer models with similar thickness are considered, the difference is significant only for the SsC, with a slightly lower DP compared to the multilayer geometry. Divergence of DP between SsC and OB above only the $630 \mathrm{~nm}$ cutoff can be explained by the increasing penetration depth with wavelength. For blue to orange-red light (440 to $610 \mathrm{~nm}$ ), the influence of deep layers on the DP is lower than that for red light (above $630 \mathrm{~nm}$ ) [Fig. 5(b)]. Indeed, photons are more likely to be absorbed into the LII than into UGL, because optical absorption in LII is higher than that in the UGL, resulting in a longer DP in the OB. The MCS demonstrates the influences of both anatomy (thickness of layers) and physiology (optical properties) on the DP, thus underlining the need to calculate specific DP on the basis of the studied brain structure and the wavelengths used. A priori assumption of DP in multilayer models is not straightforward, because nonlinear effects of both absorption and scattering combine. Increase in absorption leads obviously to a decrease in the DP but can be balanced by scattering effects.

The effects of optical parameter variations on DP were thoroughly investigated in this study to test the assumption of constant path length during activation. Regarding variations of optical coefficients, $a+10 \%$ change in the absorption coefficient corresponding approximately to a $\Delta[\mathrm{HbT}]$ of $+10 \%$ during simulated stimulation is relevant, considering the variation of total hemoglobin concentration obtained by derivation from

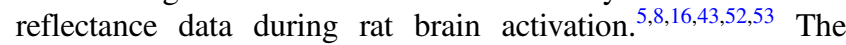
increase in absorption had no significant effects on DP below $670 \mathrm{~nm}$ and only slightly decreased the DP for higher wavelengths. Again, the effect was significant for the highest wavelengths, owing to lower absorption of blood and higher path length at these wavelengths [Fig. 3(a)]. Scattering changes also occur during activation and have been shown to participate to reflectance changes in vitro, ${ }^{54-58}$ and in vivo ${ }^{59,60}$ Several phenomena are involved in scattering changes during activation: cellular and mitochondrial swelling, ${ }^{61}$ membrane conformation changes, ${ }^{54}$ alterations of neuronal and glial structure, ${ }^{57,62}$ and blood flow changes. ${ }^{60}$ Scattering properties depend not only on the osmolarity that affects the optical index of the cytoplasm but also on the scattering properties of cytoplasmic macromolecules and organelles such as mitochondria. Lipid walls of membranes also contribute significantly to the scattering properties of tissues. The complexity of the interdependent scattering actors explains why no quantitative estimations of scattering coefficient variations in brain tissues during activation have been published yet, although a significant number of studies have been conducted in vivo and in vitro to elucidate these phenomenon since the seminal work of Hill and Keynes. ${ }^{63}$ In the present MCS study, scattering coefficients changes of $+10 \%$ or $-10 \%$ were considered, although a theoretical Mie calculation taking into consideration the increases in mitochondria and nerve diameter suggests that these variations might be much stronger. Both increases and decreases were simulated following observations made in nerve preparation ${ }^{64}$ or living tissue slices. ${ }^{58,61}$ Interpretation of the effect on DP of scattering changes is not straightforward, because increases in the scattering coefficient translates to non linear effects: decreases in the step length between interactions and increases in the albedo. As a consequence, both increases and decreases in the scattering coefficient led to a significant decrease in DP for red and/or IR wavelengths, with a slightly more pronounced effect of scattering increase [Figs. 3(b) and 3(c)]. Published data regarding anisotropy factor changes during activation are even scarcer than quantitative data on the scattering coefficient modifications. In our simulations, a 5\% increase of the anisotropy factor was considered to account for the increase of forward scattering due to increases in the scatterer dimensions. The Mie calculation suggests that this increase might be well below $5 \%$, but in the absence of measured data, this value was chosen to strengthen the potential effects of anisotropy change during activation. Assuming a local variation of $g$ (i.e., only in GL), there was no influence on DP. On the contrary, when a global change of $g$ was simulated, the DP increased along the whole range of wavelengths. If such global changes were to occur, however, they would translate to a large decrease in the reflected light, much larger than that observed in real in vivo experiments where it rarely exceeds $3 \%$ following activation. Therefore, a global change in anisotropy is unlikely to occur within millimeters of brain tissues. MCS allowed us to question the assumption of a constant DP during activation. Changes in DP remain non significant for wavelengths below $630 \mathrm{~nm}$, with the exception of global changes in anisotropy, which are unlikely to occur in non-experimental, real situations. Above $630 \mathrm{~nm}$, changes in both absorption and scattering led to significant changes in DP. Thus the assumption of a constant DP during activation is not valid for these wavelengths. Overall, the MCS results justify that multispectral activation studies in the rat brain should be carried out at wavelengths below $630 \mathrm{~nm}$. For these wavelengths, (1) the influence of the DP is insensitive to the sophistication of the model geometry, but care should be taken to define realistic depths for probed tissues; and (2) the hypothesis of constant DP during activation is true.

MCSs were carried out with models in which a $50-\mu$ m-thick bone layer was studied (Fig. 4). They showed a significant increase in DP along the whole range of wavelengths. These results are consistent with multispectral IOSI recordings in a thinned-skull preparation in guinea pigs. ${ }^{65}$ In the study by Sakaguchi et al., although the reflectance variations were about $40 \%$ less in the thinned bone preparation compared to exposed cortex, the authors managed to derive normalized hemodynamic changes similar to those observed when the cortex was exposed. Increase of the path length was attributed to high scattering and low absorption of bone. In addition, a light pipe effect was 
suggested on the basis of total reflection occurring at bone-air and bone-tissue interfaces.

Here we report the first in vivo multispectral recordings during $\mathrm{OB}$ activation. For all wavelengths, a decrease in tissue reflectance was observed following activation [Fig. 7(a)]. This observation is consistent with previous studies, most of which were carried out at $630 \mathrm{~nm}^{9,11,66}$ with the exception of the study by Meister and Bonhoeffer ${ }^{10}$ which was carried out at $707 \mathrm{~nm}$ with preliminary measurements at 546, 620, and $830 \mathrm{~nm}$. Surprisingly, there are no reports on dynamic spectral measurements in the $\mathrm{OB}$, although this approach has been applied successfully to the cortices in rats, ${ }^{8,22,52}$ mice $^{23}$ and guinea pigs. ${ }^{29}$ Derivation of hemodynamic parameters from our experiments using the modified Beer-Lambert Law showed the same activation patterns in all trials in all animals. Odorinduced activation translates into a large increase in $[\mathrm{HbR}]$ with a simultaneous moderate increase in $[\mathrm{HbT}]$ and a transient decrease in $\left[\mathrm{HbO}_{2}\right]$. These patterns were found to be independent of the wavelength choice in the analysis, which slightly modified the amplitudes of the changes but did not distort the global patterns (data not shown). On the contrary, the incorporation of a wavelength- dependent DP correction significantly altered the amplitude and time course of hemodynamic signals derived from reflectance measurements compared to analysis with no DP correction. This finding is in agreement with those in previous studies of the $\mathrm{SsC}^{43}$ and auditory cortex. ${ }^{29}$ Our study demonstrates that considering no wavelength dependence of the path length leads to strong over- or underestimation of the amplitudes of changes in $[\mathrm{HbT}]$ and $[\mathrm{HbR}]$ and may reverse the sign of $\left[\mathrm{HbO}_{2}\right]$ changes. The influence of considering a multilayer or monolayer model of OB has less significant effects. By using the lowest residuals of the fit to experimental data as a quantitative indicator of fit quality, we chose the OB multilayer model as the reference model for further studies.

Assuming a constant "scatter loss" factor throughout the experiment allows elimination of $G$ from Eq. (1) in Eq. (3). Strictly speaking, this assumption, although common, $, 814,16,67$ is not true, because scattering properties changes are likely to occur following activation. Consequently, in some previous studies, $G$ was included as a variable in the least mean squares fit. $^{23,45}$ In Ref. 23, although inclusion of this term did improve the fit quality, it did not significantly modify the time courses and amplitudes of hemoglobin concentration changes, and the authors concluded (p. 3761, last sentence of section 3) that "no indication[s] for scattering were found". Contrarily, in Ref. 45 a significant contribution of scattering changes to absorbance changes was found (Figs. 5 and 6 in Ref. 45), yet the contribution at $600 \mathrm{~nm}$ of scattering effect to optical density changes are lower than those attributed to changes in chromophore concentrations. A fit of our experimental data following olfactory stimulation was performed with $\Delta G, \Delta \mathrm{HbO}$, and $\Delta \mathrm{Hbr}$ as fitting parameters. Fitting with $\Delta G$ did not significantly change the quality of the fit, but resulted in erratic time courses for all three parameters. No indication of a scattering signal was found, similarly to the results reported previously in the barrel cortex. ${ }^{23}$ Regarding the hemodynamics changes derived using the multilayer OB model [Fig. 7(b)], the moderate and sustained increase of total blood volume during activation matches well with experimental data reported in the $\mathrm{SsC},{ }^{9,12,22,67}$ the whisker barrel cortex ${ }^{13}$ and the auditory cortex. ${ }^{28}$ The increase in HbR concentration and the simultaneous decrease in $\mathrm{HbO}_{2}$ is more surprising, though similar patterns have been reported in the
$\mathrm{SsC}$ in mice. ${ }^{22}$ Furthermore, this finding is consistent with direct partial pressure of oxygen $\left(\mathrm{PO}_{2}\right)$ measurements in the olfactory glomeruli that showed transient decreases in $\mathrm{PO}_{2}$ during activation in the glomeruli. ${ }^{68,69}$ Further studies are required to confirm and elucidate these findings. One major issue is the knowledge of the volume of tissues that are probed by the spectral recordings, which is likely to differ in the $\mathrm{SsC}$ model and the $\mathrm{OB}$ model. In Fig. 5, it appears that the reflected photons travel through distinct layers of tissues, with a total path in the glomeruli limited to about $30 \%$ of total path length. These results have to be convolved with the optical response of the instrument to derive the actual contribution of each layer. Such a study has been conducted recently in a homogeneous model of $\mathrm{SsC}$ cortex to elucidate the influence of numerical aperture and focal depth on the spatial resolution and depth sensitivity of 2-D dimensional optical imaging of the brain..$^{70}$ The authors pointed out as a conclusion the necessity to estimate the contribution of each layer to compare data across different animal models and imaging modalities. We might add that such comparisons should apply as well to different brain structures. The OB model differs significantly from the $\mathrm{SsC}$ model because hemodynamic changes are superficial in depth and highly localized in the glomerular layer, which presents a high density of capillaries, whereas activation occurs within a cortical column of several millimeters in the SsC. Such anatomofunctional differences were raised ${ }^{22}$ to explain the opposite signs of $\left[\mathrm{HBO}_{2}\right]$ and $[\mathrm{HbR}]$ changes in rats and mice during somatosensory activation and might apply as well for the OB signals reported in our study.

\section{Conclusion}

MCSs have shown that DP is relatively insensitive to the geometrical model for wavelengths below $630 \mathrm{~nm}$. For wavelengths below $630 \mathrm{~nm}$, DP in the OB was found to be insensitive to realistic changes of absorption, scattering and anisotropy. MCSs have also shown that multispectral imaging of $\mathrm{OB}$ activation through thinned bone is possible but requires a specific DP correction due to high scattering and low absorbance of bone. Multispectral data have been recorded using a fast multispectral apparatus based on a digital mirror light source at a rate of three images per wavelength per second using eight wavelengths. The same hemodynamic patterns were observed repeatedly following sensory activation of the OB: a moderate increase in total blood volume, with a simultaneous strong increase of in HbR and a small decrease of in $\mathrm{HbO}_{2}$ concentrations. Furthermore, the general tendencies of the hemodynamic parameter time courses were globally similar, regardless of whatever the model used, provided that wavelength-dependent DP was incorporated. On the basis of the residual average magnitude of the fit, the multilayer OB DP was chosen as a reference model for further studies. The observed activation pattern is consistent with recent 2-photon microscopy $\mathrm{PO}_{2}$ measurements in $\mathrm{OB}$, although they differ from what is considered a typical hemodynamic activation pattern in the $\mathrm{SsC}$. Further studies are definitely required to elucidate whether these differences are due predominantly to physiological differences or to differences in the anatomy of the SsC and OB.

\section{Acknowledgments}

This work was initiated with the support of the BQR program at Université Paris-Sud Orsay. It is currently supported by ANR Grant "Neuroenergetics." PhD scholarship of R. Renaud is funded by the French Ministry of Education. 


\section{References}

1. A. Zepeda, C. Arias, and F. Sengpiel, "Optical imaging of intrinsic signals: recent developments in the methodology and its applications," J. Neurosci. Methods 136(1),1-21 (2004).

2. T. C. Harrison, A. Sigler, and T. H. Murphy, "Simple and cost-effective hardware and software for functional brain mapping using intrinsic optical signal imaging," J. Neuros. Methods 182(2), 211-218 (2009).

3. R. D. Frostig et al., "Cortical functional architecture and local coupling between neuronal activity and the microcirculation revealed by in vivo high-resolution optical imaging of intrinsic signals," Proc. Natl. Acad. Sci. U.S.A. 87(16), 6082-6086 (1990).

4. A. Grinvald et al., "High-resolution optical imaging of functional brain architecture in the awake monkey," Proc. Natl. Acad. Sci. U.S.A. 88(24), 11559-11563 (1991).

5. J. Mayhew et al., "Spectroscopic analysis of neural activity in brain: increased oxygen consumption following activation of barrel cortex," Neuroimage 12(6), 664-675 (2000)

6. J. Mayhew et al., "Increased oxygen consumption following activation of brain: theoretical footnotes using spectroscopic data from barrel cortex," Neuroimage 13(6), 975-987 (2001).

7. A. Devor et al., "Coupling of total hemoglobin concentration, oxygenation, and neural activity in rat somatosensory cortex," Neuron 39(2), 353-359 (2003).

8. A. K. Dunn et al., "Spatial extent of oxygen metabolism and hemodynamic changes during functional activation of the rat somatosensory cortex," Neuroimage 27(2), 279-290 (2005).

9. B. D. Rubin and L. C. Katz, "Optical imaging of odorant representations in the mammalian olfactory bulb," Neuron 23(3), 499-511 (1999).

10. M. Meister and T. Bonhoeffer, "Tuning and topography in an odor map on the rat olfactory bulb," J. Neurosci. 21(4), 1351-1360 (2001).

11. H. Gurden, N. Uchida, and Z. F. Mainen, "Sensory-evoked intrinsic optical signals in the olfactory bulb are coupled to glutamate release and uptake," Neuron 52(2), 335-345 (2006).

12. J. Martindale et al., "Long duration stimuli and nonlinearities in the neural-haemodynamic coupling," J. Cereb. Blood Flow Metab. 25(5), 651-661 (2005).

13. J. Berwick et al., "Neurovascular coupling investigated with two-dimensional optical imaging spectroscopy in rat whisker barrel cortex," Eur. J. Neurosci. 22(7), 1655-1666 (2005).

14. A. Devor et al., "Coupling of the cortical hemodynamic response to cortical and thalamic neuronal activity," Proc. Natl. Acad. Sci. U.S.A. 102(10), 3822-3827 (2005).

15. J. Berwick et al., "Fine detail of neurovascular coupling revealed by spatiotemporal analysis of the hemodynamic response to single whisker stimulation in rat barrel cortex," J. Neurophysiol. 99(2), 787-798 (2008).

16. M. B. Bouchard et al., "Ultra-fast multispectral optical imaging of cortical oxygenation, blood flow, and intracellular calcium dynamics," Opt. Exp. 17(18), 15670-15678 (2009).

17. F. Pain, B. L'Heureux, and H. Gurden, "Visualizing odor representation in the brain: a review of imaging techniques for the mapping of sensory activity in the olfactory glomeruli," Cell Mol. Life Sci. $68(16)$ 2689-2709 (2011).

18. L. Belluscio et al., "Odorant receptors instruct functional circuitry in the mouse olfactory bulb," Nature 419(6904), 296-300 (2002).

19. M. Cope et al., "Methods of quantitating cerebral near infrared spectroscopy data," Adv. Exp. Med. Biol. 222, 183-189 (1988).

20. D. T. Delpy et al., "Estimation of optical pathlength through tissue from direct time of flight measurement," Phys. Med. Biol. 33(12), 1433-1442 (1988).

21. S. A. Sheth et al., "Linear and nonlinear relationships between neuronal activity, oxygen metabolism, and hemodynamic responses," Neuron 42(2), 347-355 (2004).

22. N. Prakash et al., "Temporal profiles and 2-dimensional oxy-, deoxy-, and total-hemoglobin somatosensory maps in rat versus mouse cortex," Neuroimage 37(Suppl 1), S27-S36 (2007).

23. M. Kohl et al., "Physical model for the spectroscopic analysis of cortical intrinsic optical signals," Phys. Med. Biol. 45(12), 3749-3764 (2000).

24. C. Martin et al., "Investigating neural-hemodynamic coupling and the hemodynamic response function in the awake rat," Neuroimage 32(2), 33-48 (2006).
25. B. L'Heureux, H. Gurden, and F. Pain, "Autofluorescence imaging of NADH and flavoproteins in the rat brain: insights from Monte Carlo simulations," Opt. Express 17(12), 9477-9490 (2009).

26. C. C. Petersen, "The functional organization of the barrel cortex," Neuron 56(2), 339-355 (2007).

27. J. S. McCasland and T. A. Woolsey, "High-resolution 2-deoxyglucose mapping of functional cortical columns in mouse barrel cortex," J. Comp. Neurol. 278(4), 555-569 (1988).

28. Laser Photomedicine and Biomedical Optics at the Oregon Medical Laser Center. <http://omlc.ogi.edu/>.

29. K. Sakaguchi et al., "Experimental prediction of the wavelengthdependent path-length factor for optical intrinsic signal analysis," Appl. Opt. 46(14), 2769-2777 (2007).

30. A. N. Yaroslavsky et al., "Optical properties of selected native and coagulated human brain tissues in vitro in the visible and near infrared spectral range," Phys. Med. Biol. 47(12), 2059-2073 (2002).

31. F. P. Bolin et al., "Refractive index of some mammalian tissues using a fiber optic cladding method," Appl. Opt. 28(12), 2297-2303 (1989).

32. M. Sato et al., "In vivo rat brain measurements of changes in signal intensity depth profiles as a function of temperature using wide-field optical coherence tomography," Appl. Opt. 49(30), 5686-5696 (2010).

33. I. Nishidate, K. Yoshida, and M. Sato, "Changes in optical properties of rat cerebral cortical slices during oxygen glucose deprivation," Appl. Opt. 49(34), 6617-6623 (2010).

34. S. A. Prahl et al., "A Monte Carlo model of light propagation in tissue," in SPIE Proc. Dosim. Laser Radiat. Med. Biol., vol. IS5, pp. 102-111, G. J. Müller and D. H. Sliney, Eds., SPIE Opt. Eng. Press, Bellingham, WA (1989).

35. L. Wang, S. L. Jacques, and L. Zheng, "MCML—Monte Carlo modeling of light transport in multi-layered tissues," Comput. Methods Programs Biomed. 47(2), 131-146 (1995).

36. M. Firbank et al., "Measurement of the optical properties of the skull in the wavelength range 650-950 nm," Phys. Med. Biol. 38(4), 503-510 (1993).

37. E. A. Genina, A. N. Bashkatov, and V. V. Tuchin, "Optical clearing of cranial bone," Adv. Opt. Technol. 2008, 1-8 (2008).

38. A. Edelstein et al., "Computer control of microscopes using $\mu$ Manager," in Current Protocols in Molecular Biology, Chapter 14, Unit 20, F. M. Ausubel et al., Eds., John Wiley \& Sons, London (2010).

39. R. Renaud et al., "Multispectral imaging of the olfactory bulb activation: influence of realistic differential pathlength correction factors on the derivation of oxygenation and total hemoglobin concentration maps," Proc SPIE 7902, 790208 (2011).

40. R. Chery et al., "Imaging odor-evoked activities in the mouse olfactory bulb using optical reflectance and autofluorescence signals," J. Vis. Exp. 336 (2011).

41. J. Mayhew et al., "Spectroscopic analysis of changes in remitted illumination: the response to increased neural activity in brain," Neuroimage 10(3), 304-326 (1999).

42. E. Shtoyerman et al., "Long-term optical imaging and spectroscopy reveal mechanisms underlying the intrinsic signal and stability of cortical maps in V1 of behaving monkeys," J. Neurosci. 20(21), 8111-8121 (2000).

43. U. Lindauer et al., "No evidence for early decrease in blood oxygenation in rat whisker cortex in response to functional activation," Neuroimage 13(6), 988-1001 (2001).

44. B. M. Ances, "Coupling of changes in cerebral blood flow with neural activity: what must initially dip must come back up," J. Cereb. Blood Flow Metab. 24(1), 1-6 (2004).

45. C. Sato, M. Nemoto, and M. Tamura, "Reassessment of activity-related optical signals in somatosensory cortex by an algorithm with wavelength-dependent path length," Jpn. J. Physiol. 52(3), 301-312 (2002).

46. M. Hiraoka et al., "A Monte Carlo investigation of optical pathlength in inhomogeneous tissue and its application to near-infrared spectroscopy," Phys. Med. Biol. 38(12), 1859-1876 (1993).

47. Y. Hoshi et al., "Reevaluation of near-infrared light propagation in the adult human head: implications for functional near-infrared spectroscopy," J. Biomed. Opt. 10(6), 064032 (2005).

48. E. Okada et al., "Theoretical and experimental investigation of the influence of frontal sinus on the sensitivity of the NIRS signal in the adult head," Adv. Exp. Med. Biol. 662, 231-236 (2010). 
49. E. Okada et al., "Theoretical and experimental investigation of nearinfrared light propagation in a model of the adult head," in $A d v \operatorname{Exp}$ Med Biol Book Series, Oxygen Transport to Tissue XXXI, Chapter 2, pp. 231-236, E. Takahashi and D. F. Bruley, Eds., Springer, Berlin (1997).

50. M. Firbank, E. Okada, and D. T. Delpy, "Investigation of the effect of discrete absorbers upon the measurement of blood volume with nearinfrared spectroscopy," Phys. Med. Biol. 42(3), 465-477 (1997).

51. A. Talsma, B. Chance, and R. Graaff, "Corrections for inhomogeneities in biological tissue caused by blood vessels," J. Opt. Soc. Am. A Opt. Image Sci. Vis. 18(4), 932-939 (2001).

52. M. Jones et al., "Concurrent optical imaging spectroscopy and laserDoppler flowmetry: the relationship between blood flow, oxygenation, and volume in rodent barrel cortex," Neuroimage 13(6), 1002-1015 (2001).

53. E. M. C. Hillman et al., "Depth-resolved optical imaging and microscopy of vascular compartment dynamics during somatosensory stimulation," Neuroimage 35(1), 89-104 (2007).

54. L. B. Cohen, R. D. Keynes, and B. Hille, "Light scattering and birefringence changes during nerve activity," Nature 218(5140), 438-441 (1968).

55. P. G. Aitken et al., "Use of intrinsic optical signals to monitor physiological changes in brain tissue slices," Methods 18(2), 91-103 (1999).

56. R. D. Andrew, C. R. Jarvis, and A. S. Obeidat, "Potential sources of intrinsic optical signals imaged in live brain slices," Methods 18(2), 185-196 (1999).

57. C. R. Jarvis et al., "Interpretation of intrinsic optical signals and calcein fluorescence during acute excitotoxic insult in the hippocampal slice," Neuroimage 10(4), 357-372 (1999).

58. D. Fayuk et al., "Two different mechanisms underlie reversible, intrinsic optical signals in rat hippocampal slices," J. Neurophysiol. 87(4), 1924-1937 (2002).
59. D. M. Rector et al., "Spatio-temporal mapping of rat whisker barrels with fast scattered light signals," Neuroimage 26(2), 619-627 (2005).

60. M. Tomita, "Flow effect impacts NIRS, jeopardizing quantification of tissue hemoglobin," Neuroimage 33(1), 13-16 (2006).

61. L. J. Johnson et al., "Optical scatter imaging detects mitochondrial swelling in living tissue slices," Neuroimage 17(3), 1649-1657 (2002).

62. E. Syková et al. and S. Prokopová-Kubinová, "Extracellular space diffusion and pathological states," Prog. Brain Res. 125, 155-178 (2000).

63. D. K. Hill and R. D. Keynes, "Opacity changes in stimulated nerve," J. Physiol. (Lond.) 108(3), 278-281 (1949).

64. L. B. Cohen, B. Hille, and R. D. Keynes, "Changes in axon birefringence during the action potential," J. Physiol. (Lond.) 211(2), 495-515 (1970).

65. K. Sakaguchi et al., "Influence of a skull cranial window on the measurement of haemoglobin concentration in cortical tissue by multispectral imaging analysis," Opt. Rev. 16(2), 74-80 (2010).

66. N. Uchida and Z. F. Mainen, "Speed and accuracy of olfactory discrimination in the rat," Nat. Neurosci. 6(11), 1224-1229 (2003).

67. A. Devor et al., "Stimulus-induced changes in blood flow and 2deoxyglucose uptake dissociate in ipsilateral somatosensory cortex," J. Neurosci 28(53), 14347-14357 (2008).

68. J. Lecoq et al., "Odor-evoked oxygen consumption by action potential and synaptic transmission in the olfactory bulb," J. Neurosci. 29(5), 1424-1433 (2009).

69. J. Lecoq et al., "Simultaneous two-photon imaging of oxygen and blood flow in deep cerebral vessels," Nat. Med. 17(7), 893-898 (2011).

70. P. Tian et al., "Monte Carlo simulation of the spatial resolution and depth sensitivity of two-dimensional optical imaging of the brain," J. Biomed. Opt. 16(1), 016006 (2011). 